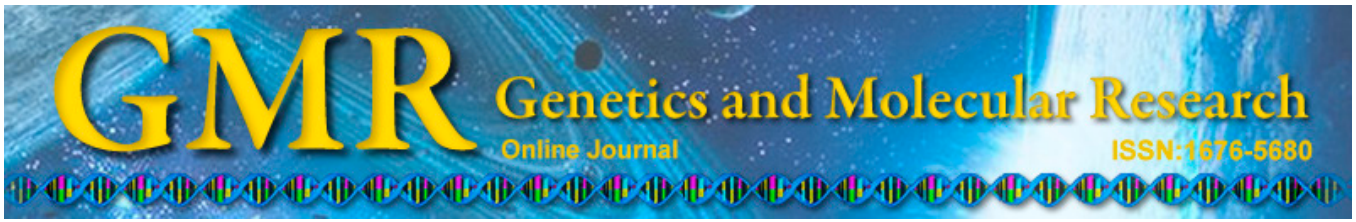

\title{
Deciphering the spectrum of somatic mutations in the entire mitochondrial DNA genome
}

\author{
X.Z. Chen ${ }^{1}$, Y. Fang ${ }^{2}$, Y.H. Shi ${ }^{3}$, J.H. Cui ${ }^{4}$, L.Y. Li ${ }^{5}$, Y.C. Xu' ${ }^{5}$ and B. Ling ${ }^{1}$ \\ ${ }^{1}$ Department of Intensive Care Unit, \\ The Second People's Hospital of Yunnan Province, \\ Kunming, Yunnan Province, China \\ ${ }^{2}$ Department of Anesthesiology, \\ The First Affiliated Hospital of Kunming Medical University, Kunming, \\ Yunnan Province, China \\ ${ }^{3}$ Department of Clinical Laboratory, Shanxi Tumor Hospital, Taiyuan, Shanxi, \\ China \\ ${ }^{4}$ Department of Anatomy, Medical Training Center of Shenyang Command, \\ PLA, Dalian, Liaoning, China \\ ${ }^{5}$ Department of Cardiology and Clinical Laboratory, 211 Hospital of PLA, \\ Harbin, Heilongjiang, China \\ Corresponding author: B. Ling \\ E-mail: binlingre@163.com
}

Genet. Mol. Res. 14 (2): 4331-4337 (2015)

Received May 13, 2014

Accepted October 22, 2014

Published April 30, 2015

DOI http://dx.doi.org/10.4238/2015.April.30.5

\begin{abstract}
The mitochondrion is a crucial intracellular organelle responsible for regulating cellular energy metabolism, producing free radicals, initiating and executing the apoptotic pathways. Previous studies have shown that somatic mutations in mitochondrial DNA are associated with various tumors, which may be involved during carcinogenesis and tumor progression. To examine the mutation pattern in cancer, 625 reported somatic mutations in the mitochondrial DNA genome were analyzed. We found that, except for deletions and insertions, most somatic mutations were point mutations, accounting for $89.44 \%$ of somatic mutations. Transition was the predominant form of
\end{abstract}


somatic mutation in the entire mitochondrial DNA genome, accounting for $87.12 \%$ of point mutations, most of which were homoplastic. Frequency statistics analysis of point mutations indicated that, except for 3 tRNA genes, the mutations were distributed on all resting genes and in the D-loop region, with the latter showing the highest frequency of somatic mutation (19.34\%), followed by the tRNA leucine 2 gene and non-coding regions between base pairs 5892 and 5903, while 13 coding-region genes and 2 rRNA genes showed a relatively lower frequency of somatic point mutations. Nonsynonymous mutations and terminal amino acid changes were the primary point somatic mutations detected from 13 coding-region genes, which may cause mitochondrial dysfunction in cancer cells. We found that the somatic mutations may affect the mitochondrial DNA genome; the non-coding region should be examined to identify somatic mutations as potential diagnostic biomarkers for early detection of cancer.

Key words: Cancer; Mitochondrial DNA genome; Somatic mutation

\section{INTRODUCTION}

The mitochondrion is a membrane-bound organelle present in most eukaryotic cells. It plays essential generating roles in energy production through oxidative phosphorylation, free radical formation, regulation and execution of apoptosis, and conversion of various metabolic intermediates (Wallace, 1994). Mitochondrial DNA (mtDNA) is composed of an autonomously replicating DNA genome composed of 16,569 bp and encodes 37 genes, including $12 \mathrm{~S}$ and 16S rRNAs, 22 tRNAs, and 13 essential subunits used in oxidative phosphorylation (Anderson et al., 1981). The genome lacks protective histones and has an extremely inefficient DNA mismatch repair mechanism (Croteau and Bohr, 1997); it also exhibits high levels of reactive oxygen species (ROS), generated through oxidative phosphorylation in this organelle. ROS can damage mtDNA and cause mutations (Yakes and Van Houten, 1997), making the genome vulnerable to oxidative or other genotoxic damages, resulting in a much higher mutation rate (10- to 200-fold) than in nuclear DNA (nDNA) (Brown et al., 1979; Larsen et al., 2005), which is one of the major characteristics of mtDNA.

mtDNA sequence variations progressively accumulate in mtDNA genomes, increasing the mutations in germ line cells of females, which can be transmitted to subsequent generations. These mutations form the maternal backgrounds of an individual and can affect the expression of different diseases (Fuku et al., 2007; Ji et al., 2008). Mutations in other cells in the body, except germ cells, are not passed to next generation, but can cause dysfunction in mtDNA genomes and result in various diseases (Zanssen and Schon, 2005; Chatterjee et al., 2006), including bladder cancer (Fliss et al., 2000; Guney et al., 2012), head and neck cancer (Mizumachi et al., 2008), lung cancer (Fliss et al., 2000; Jin et al., 2007; Fang et al., 2015), pancreatic cancer (Navaglia et al., 2006), hepatocellular carcinomas (Okochi et al., 2002), colorectal cancer (Feng et al., 2012), thyroid cancer (Abu-Amero et al., 2005), breast cancer (Cai et al., 2011; Fendt et al., 2011; Alhomidi et al., 2013), prostate cancer (Parr et al., 2006; Ashtiani et al., 2012), and gastric carcinomas (Hung et al., 2010; Bi et al., 2011). Somatic mutation may arise from oxidative damage caused by the accumulation of ROS in tissues and the deficiency of mtDNA repair 
systems (Croteau and Bohr, 1997; Yakes and Van Houten, 1997). The role of somatic mutation in carcinogenesis remains controversial; however, considering the relatively higher frequency of somatic mutations in tumor tissues detected through comparison with matched normal tissue, somatic mutations may be useful as biomarkers of carcinogenesis (Sui et al., 2006). However, the most reported somatic mutations were identified in the control region of the mtDNA genome because of its much higher mutation rates (Yu, 2012). Some studies included a limited sample size and did not sequence the entire mtDNA genome (Wang and Zhao, 2011; Fang et al., 2015), and thus were not sufficient to determine the somatic mutation spectrum in the entire mtDNA genome. Therefore, identifying the spectrum of somatic mutations in the entire mtDNA genome may increase the understanding of the role of mtDNA mutations in carcinogenesis and be useful for identifying diagnostic biomarkers for early detection of cancer.

In this study, 625 somatic mutations in the entire mtDNA genome of different diseases from MITOMAP (Ruiz-Pesini et al., 2007) were analyzed.

\section{MATERIAL AND METHODS}

\section{Materials}

A total of 625 somatic mutations covering the entire mtDNA genome were retrieved from MITOMAP (Ruiz-Pesini et al., 2007), which was updated in October, 2013. Somatic mutations in the overlapping fragment of the ATP 6 and ATP 8 genes were considered to be 2 different mutations. The somatic mutations were present in tumor tissue, including Twinkle/ progressive external ophthalmoplegia (PEO) eye muscle, skeletal muscle, polymerase gamma/ PEO muscle, and mitochondrial neurogastrointestinal encephalomyopathy fibroblasts, among others. Previous genetic studies only sequenced the D-loop region of mtDNA, which accounts for $6.77 \%$ of the entire mtDNA genome, or were limited by relatively small sample sizes in examining the entire mtDNA genome. Thus, to comprehensively determine the general pattern of somatic mutations in the entire mtDNA genome, we recorded the position of each somatic mutation rather than the frequency.

\section{Methods}

Somatic mutations were classified as point mutations, deletions, or insertions according to previous studies (Li and Hong, 2012; Yu, 2012). The spectrum of somatic mtDNA mutations in common primary human malignancies and other diseases were calculated. The changing format for the base pairs with somatic mutations in the entire mtDNA genome were identified, and their frequencies were calculated and compared between mtDNA genes, including $12 \mathrm{~S}$ and $16 \mathrm{~S}$ rRNA, 22 tRNAs, non-coding regions, and 13 protein-coding region genes (Anderson et al., 1981; Andrews et al., 1999).

\section{RESULTS AND DISCUSSION}

As shown in Table S1, in total, 625 somatic mutations were analyzed in this study, including point mutations, insertions, and deletions. Point mutations accounted for $84.64 \%$ (529/625) of all somatic mutations in the entire mtDNA genome, which was much higher than deletions $(6.24 \% ; 39 / 625)$ and insertions $(4.32 \% ; 27 / 625)$ (Table S1). Most deletions and in- 
sertions in the gene-coding region resulted in frameshifts, causing dysfunction in the mtDNA genomes. This may affect the initial phase of tumorigenesis, particularly clonal expansion during tumor progression (Yu, 2012), although whether insertions in the mtDNA control region are related to tumor initiation and development remain controversial, as insertions have also been detected in healthy populations (Hung et al., 2008).

Because point mutations accounted for approximately $84.64 \%$ of all somatic mutations in the entire mtDNA genome, we further analyzed these mutations. We analyzed 559 point somatic mutations and found that the transition of base pairs between adenine (A) and guanine $(\mathrm{G})$, as well as cytosine $(\mathrm{C})$ and thymine $(\mathrm{T})$ accounted for $87.30 \%(488 / 559)$ of all point mutations. Further, we conducted statistical analysis on the status of the base pairs for the point somatic mutations, except for 52 somatic mutations lacking information regarding the status of the base pairs. We found that the most frequent $(52.48 \%)$ of the somatic point mutations were homoplasmic, and $45.76 \%$ were heterogenetic, which agrees with the results of a previous study (Li and Hong, 2012; Yu, 2012) and the fact that cell fission with somatic mtDNA mutations increase the number of and fix mutant types in tumor tissue (Polyak et al., 1998; Penta et al., 2001). We also found that most point mutations were transitions between pyridine and purines $(12.70 \% ; 71 / 559)$; this mutation pattern was similar to that of oxidative decay on DNA caused by ROS in normal tissues, and may explain the elevated levels of ROS during the tumor development (Beckman and Ames, 1997). We further evaluated the general pattern of somatic mutations in different genes, as well as determined which genes are more sensitive to ROS and can serve as potential biomarkers for future clinical applications. Thus, the point mutation frequency in each gene was determined according to their gene locus, and the spectrum of each gene, including 13 essential subunits of the oxidative phosphorylation system, 2 rRNAs, 22 tRNAs, and the D-loop region, were calculated. As shown in Table 1, except for 3 tRNA genes, including TRNN(TN), TRNT (TT), and TRNV (TV) without somatic mutations, the remaining 34 genes and control regions showed somatic point mutations. Specifically, the mtDNA control region showed the highest somatic mutation frequency compared to the other genes in the entire mtDNA genome, and a total of 217 somatic mutations were detected among the $1122 \mathrm{bp}(19.34 \%)$ in the mtDNA control region. The tRNA leucine 2 gene $(8.45 \%)$, non-coding region between positions $5892-5903$ (8.33\%), and tRNA serine 2 genes (5.08\%) showed higher somatic mutation frequencies; the TRNM (TM), TRNA (TA), TRNP (TP) and TRNI (TI) genes have moderate somatic mutation frequencies; however, coding region genes (including ND1, ND2, ND3, ND4, ND4L, ND5, ND6, COI, COII, COIII, ATP6, ATP8, and CYTB) and ribonucleotide reductase genes (including ribonucleotide reductase 1 and 2) showed relatively lower frequencies of somatic mutation. This indicates that the mtDNA control region is a hotspot region for somatic point mutations, and this region is much more sensitive to ROS than other genes. Thus, the mtDNA control region may serve as a biomarker for detecting somatic mutations in clinical diagnosis.

Further, because of the crucial biological role of the 13 protein-coding genes in the generation of ATP and the fact that somatic mutations in these genes can affect biological function, the somatic mutations detected in the 13 protein-coding genes were further analyzed. Among the 264 point somatic mutations of the $13 \mathrm{mtDNA}$ protein-coding region genes, except 21 terminal code caused by somatic mutations, our results indicated that non-synonymous mutations accounted for a higher frequency $(62.88 \%$; 166/264) than synonymous mutations $(37.12 \% ; 98 / 264)$; however, this pattern was quite different from that of general populations as 
shown in a previous study (Liu et al., 2011). This relaxation of negative selection may play a crucial role in the accumulation of somatic mutations (Liu et al., 2011); these mutations show a high potential to cause mitochondrial dysfunction in cancer cells (Lee et al., 2010).

Table 1. Frequency of somatic mutations in each mtDNA gene.

\begin{tabular}{|c|c|c|c|c|}
\hline Gene & Somatic mutations (N) & Position of gene (bp) & Length of gene (bp) & Frequency \\
\hline D-loop & 217 & $16024 . .16569 / 1 . .576$ & 1122 & $19.34 \%$ \\
\hline ND3 & 13 & $10059 . .10404$ & 346 & $3.76 \%$ \\
\hline ND4L & 11 & $10470 . .10766$ & 297 & $3.70 \%$ \\
\hline ND1 & 30 & $3307 . .4262$ & 956 & $3.14 \%$ \\
\hline $\mathrm{CO} 3$ & 23 & $9207 . .9990$ & 784 & $2.93 \%$ \\
\hline $\mathrm{CO} 1$ & 38 & $5904 . .7445$ & 1542 & $2.46 \%$ \\
\hline ATP8 & 5 & $8366 . .8572$ & 207 & $2.42 \%$ \\
\hline CYB & 26 & $14747 . .15887$ & 1141 & $2.28 \%$ \\
\hline ND4 & 30 & $10760 . .12137$ & 1378 & $2.18 \%$ \\
\hline ND2 & 21 & $4470 . .5511$ & 1042 & $2.02 \%$ \\
\hline ND6 & 10 & $14149 . .14673$ & 525 & $1.90 \%$ \\
\hline ND5 & 29 & $12337 . .14148$ & 1812 & $1.60 \%$ \\
\hline $\mathrm{CO} 2$ & 10 & $7586 . .8269$ & 684 & $1.46 \%$ \\
\hline ATP6 & 15 & $8527 . .9207$ & 681 & $2.20 \%$ \\
\hline RNR1 & 13 & $648 . .1601$ & 954 & $1.36 \%$ \\
\hline RNR2 & 25 & $1671 . .3229$ & 1559 & $1.60 \%$ \\
\hline non-coding & 1 & $5892 . .5903$ & 12 & $8.33 \%$ \\
\hline TRNA (TA) & 3 & $5512 . .5579$ & 68 & $4.41 \%$ \\
\hline TRNC (TC) & 1 & $5761 . .5826$ & 66 & $1.52 \%$ \\
\hline TRND (TD) & 1 & $7518 . .7585$ & 68 & $1.47 \%$ \\
\hline TRNE (TE) & 1 & $14674 . .14742$ & 69 & $1.45 \%$ \\
\hline TRNF (TF) & 2 & $577 . .647$ & 71 & $2.82 \%$ \\
\hline TRNG (TG) & 1 & $9991 . .10058$ & 68 & $1.47 \%$ \\
\hline TRNH (TH) & 1 & $12138 . .12206$ & 69 & $1.45 \%$ \\
\hline TRNI (TI) & 3 & $4263 . .4331$ & 69 & $4.35 \%$ \\
\hline TRNK (TK) & 1 & $8295 . .8364$ & 70 & $1.43 \%$ \\
\hline TRNL1 (TL1) & 2 & $3230 . .3304$ & 75 & $2.67 \%$ \\
\hline TRNL2 (TL2) & 6 & $12266 . .12336$ & 71 & $8.45 \%$ \\
\hline TRNM (TM) & 3 & $4402 . .4469$ & 68 & $4.41 \%$ \\
\hline TRNP (TP) & 3 & $15956 . .16023$ & 68 & $4.41 \%$ \\
\hline TRNQ (TQ) & 2 & $4329 . .4400$ & 72 & $2.78 \%$ \\
\hline TRNR (TR) & 2 & $10405 . .10469$ & 65 & $3.08 \%$ \\
\hline TRNS1 (TS1) & 1 & $7446 . .7514$ & 69 & $1.45 \%$ \\
\hline TRNS2 (TS2) & 3 & $12207 . .12265$ & 59 & $5.08 \%$ \\
\hline TRNW (TW) & 2 & $5512 . .5579$ & 68 & $2.94 \%$ \\
\hline TRNY (TY) & 1 & $5826 . .5891$ & 66 & $1.52 \%$ \\
\hline
\end{tabular}

In summary, by analyzing 625 somatic mutations detected in the entire mtDNA genome, our results indicated that, except for deletions and insertions, most mtDNA somatic mutations were point mutations. Homogeneity is one of the major characteristics of point somatic mutations, most of which were transitions from A to $\mathrm{G}$, as well as $\mathrm{C}$ to $\mathrm{T}$, potentially because of the high ROS levels in the mitochondria. By determining the somatic mutation frequency of the control region and genes in each mtDNA coding region, our results indicated that the control region of the mtDNA genome has the highest somatic mutation frequency among other genes, particularly the 13 protein-coding region genes and 2 sRNA genes, which may be related to their crucial biological role. Our results indicate that the mtDNA control region can be used to detect somatic mutations as a diagnostic biomarker for cancer detection. Furthermore, most somatic point mutations in coding-region genes were non-synonymous or frame-shift mutations in the mRNA genes of the mitochondrial genome, which have high potential to cause mitochondrial dysfunction in cancer cells. 


\section{ACKNOWLEDGMENTS}

We thank the anonymous reviewers for their helpful comments on the manuscript.

\section{Supplementary material}

\section{REFERENCES}

Abu-Amero KK, Alzahrani AS, Zou M and Shi Y (2005). High frequency of somatic mitochondrial DNA mutations in human thyroid carcinomas and complex I respiratory defect in thyroid cancer cell lines. Oncogene 24: 1455-1460.

Alhomidi MA, Vedicherla B, Movva S, Rao PK, et al. (2013). Mitochondrial D310 instability in Asian Indian breast cancer patients. Tumour Biol. 34: 2427-2432.

Anderson S, Bankier AT, Barrell BG, de Bruijn MH, et al. (1981). Sequence and organization of the human mitochondrial genome. Nature 290: 457-465.

Andrews RM, Kubacka I, Chinnery PF, Lightowlers RN, et al. (1999). Reanalysis and revision of the Cambridge reference sequence for human mitochondrial DNA. Nat. Genet. 23: 147.

Ashtiani ZO, Heidari M, Hasheminasab SM, Ayati M, et al. (2012). Mitochondrial D-Loop polymorphism and microsatellite instability in prostate cancer and benign hyperplasia patients. Asian Pac. J. Cancer Prev. 13: 3863-3868.

Beckman KB and Ames BN (1997). Oxidative decay of DNA. J. Biol. Chem. 272: 19633-19636.

Bi R, Li WL, Chen MQ, Zhu Z, et al. (2011). Rapid identification of mtDNA somatic mutations in gastric cancer tissues based on the mtDNA phylogeny. Mutat. Res. 709-710: 15-20.

Brown WM, George M Jr and Wilson AC (1979). Rapid evolution of animal mitochondrial DNA. Proc. Natl. Acad. Sci. US A 76: 1967-1971.

Cai FF, Kohler C, Zhang B, Chen WJ, et al. (2011). Mutations of mitochondrial DNA as potential biomarkers in breast cancer. Anticancer Res. 31: 4267-4271.

Chatterjee AE, Mambo E and Sidransky D (2006). Mitochondrial DNA mutations in human cancer. Oncogene 25: 4663-4674

Croteau DL and Bohr VA (1997). Repair of oxidative damage to nuclear and mitochondrial DNA in mammalian cells. $J$. Biol. Chem. 272: 25409-25412.

Fang Y, Huang J, Zhang J, Wang J, et al. (2015). Detecting the somatic mutations spectrum of Chinese lung cancer by analyzing the whole mitochondrial DNA genomes. Mitochondrial DNA 26: 56-60.

Fendt L, Niederstätter H, Huber G, Zelger B, et al. (2011). Accumulation of mutations over the entire mitochondrial genome of breast cancer cells obtained by tissue microdissection. Breast Cancer Res. Treat. 128: 327-336.

Feng S, Xiong LL, Ji ZN, Cheng W, et al. (2012). Correlation between increased ND2 expression and demethylated displacement loop of mtDNA in colorectal cancer. Mol. Med. Rep. 6: 125-130.

Fliss MS, Usadel H, Cabellero OL, Wu L, et al. (2000). Facile detection of mitochondrial DNA mutations in tumors and bodily fluids. Science 287: 2017-2019.

Fuku N, Park KS, Yamada Y, Nishigaki Y, et al. (2007). Mitochondrial haplogroup N9a confers resistance against type 2 diabetes in Asians. Am. J. Hum. Genet. 80: 407-415.

Guney AI, Ergec DS, Tavukcu HH, Koc G, et al. (2012). Detection of mitochondrial DNA mutations in nonmuscle invasive bladder cancer. Genet. Test. Mol. Biomarkers 16: 672-678.

Hung WY, Lin JC, Lee LM, Wu CW, et al. (2008). Tandem duplication/triplication correlated with poly-cytosine stretch variation in human mitochondrial DNA D-loop region. Mutagenesis 23: 137-142.

Hung WY, Wu CW, Yin PH, Chang CJ, et al. (2010). Somatic mutations in mitochondrial genome and their potential roles in the progression of human gastric cancer. Biochim. Biophys. Acta 1800: 264-270.

Ji Y, Zhang AM, Jia X, Zhang YP, et al. (2008). Mitochondrial DNA haplogroups M7b1'2 and M8a affect clinical expression of leber hereditary optic neuropathy in Chinese families with the m.11778G - >a mutation. Am. J. Hum. Genet. 83: 760-768.

Jin X, Zhang J, Gao Y, Ding K, et al. (2007). Relationship between mitochondrial DNA mutations and clinical characteristics in human lung cancer. Mitochondrion 7: 347-353.

Larsen NB, Rasmussen M and Rasmussen LJ (2005). Nuclear and mitochondrial DNA repair: similar pathways? Mitochondrion 5: 89-108.

Lee HC, Chang CM and Chi CW (2010). Somatic mutations of mitochondrial DNA in aging and cancer progression. Ageing Res. Rev. 9 (Suppl 1): S47-S58.

Li H and Hong ZH (2012). Mitochondrial DNA mutations in human tumor cells. Oncol. Lett. 4: 868-872.

Liu J, Wang LD, Sun YB, Li EM, et al. (2011). Deciphering the signature of selective constraints on cancerous 
mitochondrial genome. Mol. Biol. Evol. 29: 1255-1261.

Mizumachi T, Suzuki S, Naito A, Carcel-Trullols J, et al. (2008). Increased mitochondrial DNA induces acquired docetaxel resistance in head and neck cancer cells. Oncogene 27: 831-838.

Navaglia F, Basso D, Fogar P, Sperti C, et al. (2006). Mitochondrial DNA D-loop in pancreatic cancer: somatic mutations are epiphenomena while the germline 16519 T variant worsens metabolism and outcome. Am. J. Clin. Pathol. 126: 593-601.

Okochi O, Hibi K, Uemura T, Inoue S, et al. (2002). Detection of mitochondrial DNA alterations in the serum of hepatocellular carcinoma patients. Clin. Cancer Res. 8: 2875-2878.

Parr RL, Dakubo GD, Crandall KA, Maki J, et al. (2006). Somatic mitochondrial DNA mutations in prostate cancer and normal appearing adjacent glands in comparison to age-matched prostate samples without malignant histology. $J$. Mol. Diagn. 8: 312-319.

Penta JS, Johnson FM, Wachsman JT and Copeland WC (2001). Mitochondrial DNA in human malignancy. Mutat. Res. 488: 119-133.

Polyak K, Li Y, Zhu H, Lengauer C, et al. (1998). Somatic mutations of the mitochondrial genome in human colorectal tumours. Nat. Genet. 20: 291-293.

Ruiz-Pesini E, Lott MT, Procaccio V, Poole JC, et al. (2007). An enhanced MITOMAP with a global mtDNA mutational phylogeny. Nucleic Acids Res. 35: D823-D828.

Sui G, Zhou S, Wang J, Canto M, et al. (2006). Mitochondrial DNA mutations in preneoplastic lesions of the gastrointestinal tract: a biomarker for the early detection of cancer. Mol. Cancer 5: 73.

Wallace DC (1994). Mitochondrial-DNA sequence variation in human-evolution and disease. Proc. Natl. Acad. Sci. US A 91: 8739-8746.

Wang C-Y and Zhao Z-B (2011). Somatic mtDNA mutations in lung tissues of pesticide-exposed fruit growers. Toxicology 291: 51-55.

Yakes FM and Van Houten B (1997). Mitochondrial DNA damage is more extensive and persists longer than nuclear DNA damage in human cells following oxidative stress. Proc. Natl. Acad. Sci. U S A 94: 514-519.

Yu M (2012). Somatic mitochondrial DNA mutations in human cancers. Adv. Clin. Chem. 57: 99-138.

Zanssen S and Schon EA (2005). Mitochondrial DNA mutations in cancer. PLoS Med. 2: e401. 